\title{
Comportement mécanique des glaces de glaciers
}

\author{
O. Castelnau et P. Duval \\ Laboratoire de Glaciologie et de Géophysique de l'Environnement, C.N.R.S.
}

\section{IINTRODUCTION}

Les études sur la modélisation de l'écoulement des glaciers et calottes polaires se sont développées ces dernières années. Ces recherches sont surtout motivées par le besoin de connaître la réponse des glaciers et calottes polaires à un changement climatique. Parallèlement, l'interprétation des données climatiques tirées de l'étude des glaces polaires nécessite la datation des carottes de glace. Actuellement, l'âge de ces glaces est déduit de la connaissance de l'histoire mécanique des particules de glace, elle-même obtenue par la modélisation de l'écoulement des calottes polaires. Certains repères (couches volcaniques, transitions climatiques...) permettent d'ajuster certains paramètres du modèle.

La performance des modèles d'écoulement de glace est limitée par la connaissance encore insuffisante des lois rhéologiques des glaces. A la surface des régions centrales de l'Antarctique de l'Est, la température moyenne annuelle est inférieure à $-50^{\circ} \mathrm{C}$. Ces basses températures associées à un faible taux d'accumulation, à une pente de la surface inférieure à $10^{-3}$ et à des épaisseurs de glace souvent supérieures à $3000 \mathrm{~m}$, font que les vitesses de déformation de la glace sont très faibles. A Vostok, la vitesse de déformation selon la verticale est de l'ordre de $2,5 \times 10^{-13} \mathrm{~s}^{-1}$. Ces conditions ne peuvent être convenablement reproduites en laboratoire. Ces difficultés sont toujours à l'origine de discussions sur la valeur du coefficient $n$ de sensiblité de la vitesse de déformation à la contrainte au-dessous de 0,1 MPa. Les données récentes de terrain telles celles tirées de l'inclinaison des trous de forage ou des profils de densité du névé et de la glace bulleuse ont permis de confirmer la faible valeur de $n$ aux faibles contraintes [1, 2].

Les carottages profonds réalisés en Antarctique et au Groenland ont permis de mettre en évidence des glaces dont l'anisotropie est très marquée. Celle-ci est caractérisée soit par un alignement des axes $c$ des cristaux selon la verticale $[3,4]$, soit par une répartition aléatoire des axes $c$ dans un plan vertical transverse à la direction de l'écoulement [5]. Ces deux types de fabriques, qui résultent de la rotation des cristaux par le glissement intracristallin, confèrent à la glace un comportement mécanique très différent; la viscosité de la glace correspondant à une compression selon la verticale varie de plus d'un ordre de grandeur entre les deux cas [6].

L'étude des glaces polaires a aussi permis de mettre en évidence plusieurs processus de recristallisation, les conditions de passage d'un régime de recristallisation à l'autre étant elles-mêmes bien définies $[7,8]$. Les fabriques décrites ci-dessus sont associées à des régimes de recristallisation pour lesquels la vitesse de migration des joints de grains est faible. Un régime de recristallisation associé à de grandes vitesses de migration des joints est observé dans les couches basales de la calotte glaciaire de l'Antarctique, pour des températures supérieures à $-10^{\circ} \mathrm{C}$ [3]. Cette recristallisation dynamique est d'ailleurs celle qui intervient dans les glaces des glaciers tempérés. Sous de telles conditions, l'orientation préférentielle des cristaux résulte de la recristallisation et non de la rotation des cristaux par le glissement cristallin [9].

L'effet de la température sur le comportement viscoplastique de la glace entre $-50^{\circ} \mathrm{C}$ et la température de fusion de la glace ne peut se résumer à l'activation thermique d'un seul processus physique. Les processus de recristallisation ainsi que les modes de déformation changent avec la température. Pour la glace tempérée, il faut considérer la déformation d'un matériau à deux phases. Sous l'effet de contraintes non hydrostatiques, l'eau n'est plus uniquement localisée aux jonctions de trois ou quatre grains, mais peut être aussi à la jonction de deux grains sous forme de lentilles perpendiculaires à l'axe de compression maximum [10-12]. S'agissant de la glace, roche fortement anisotrope, l'eau doit atténuer le champ de contraintes internes qui s'installe après l'application d'une charge. Elle peut aussi favoriser les processus de migration des joints de grain ou jouer le rôle de sources de dislocations.

Dans ce papier, nous présentons une analyse des mécanismes de recristallisation et de déformation qui interviennent dans les glaces des glaciers froids et tempérés. Enfin, pour ce qui concerne les glaces polaires, nous montrerons l'intérêt des approches « micro/macro », permettant le passage du monocristal au polycristal, pour décrire le comportement mécanique.

\section{RECRISTALLISATION DYNAMIQUE}

\subsection{Grossissement des grains}

La taille des grains dans le névé et la glace des couches supérieures des calottes polaires croît avec la profondeur. La force motrice de ce grossissement provient de l'énergie libre des joints de grains. La taille des grains varie linéairement avec le temps $t$ :

$$
l^{2}=l_{0}^{2}+K t \text { ( } K \text { est la vitesse de grossissement) }
$$

Le profil de la vitesse de croissance des grains le long de la carotte de $905 \mathrm{~m}$ de Dome C (Antarctique de l'Est) est donné sur la figure I. Il montre les mêmes caractéristiques que le profil isotopique, lequel traduit l'évolution de 


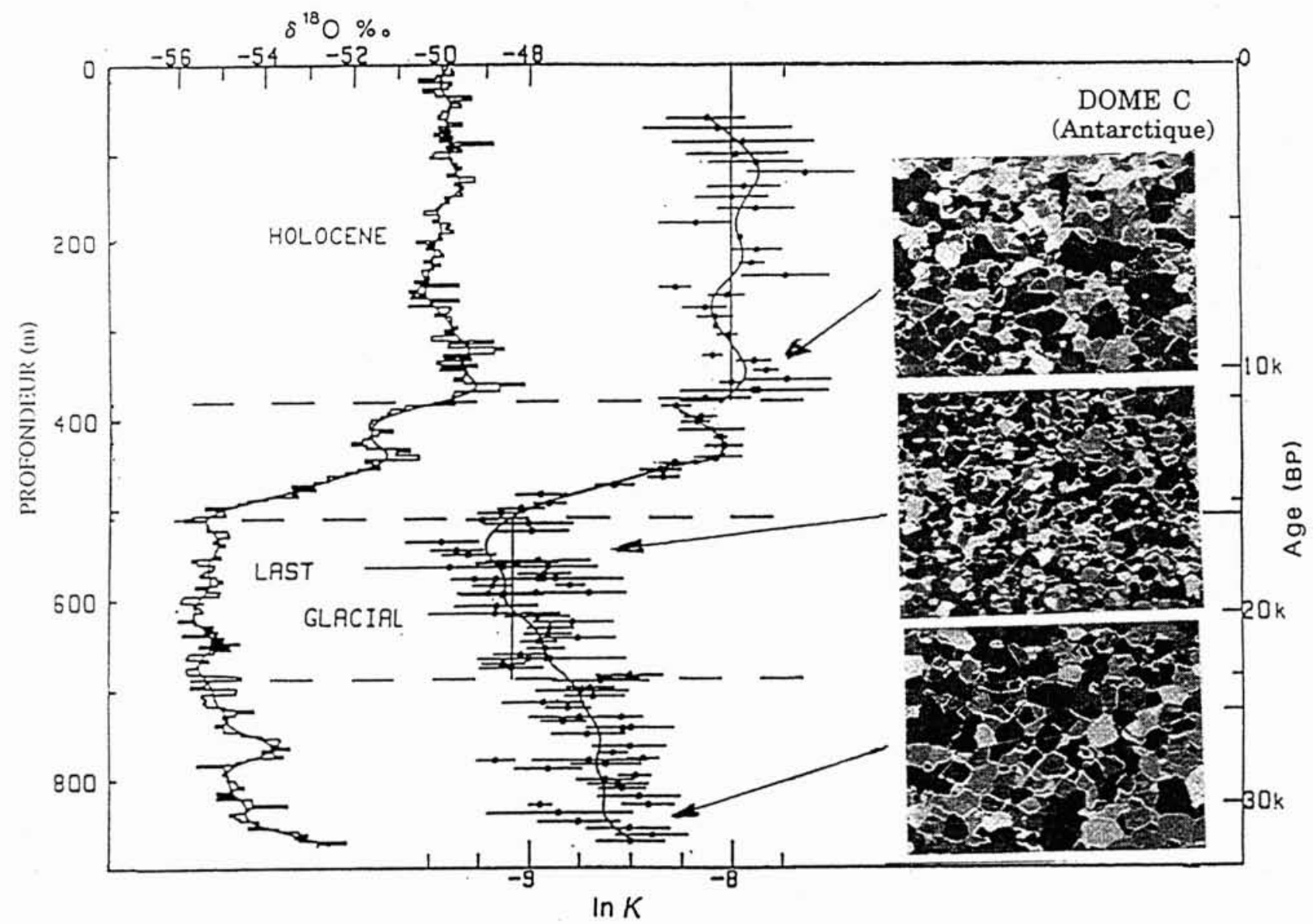

1. Variation de la teneur isotopique $\delta^{18} \mathrm{O}$ et de la vitesse de croissance des cristaux de glace en fonction de la profondeur et de l'âge de la carotte de Dôme $\mathrm{C}$ (d'après [13]) ; les photographies montrent la texture de la glace aux profondeurs : 392,$50 ; 566,10$ et $878,00 \mathrm{~m}$.

la température de surface au cours des 35000 dernières années. Petit et al. [13] font l'hypothèse que la microstructure des cristaux de glace (densité de défauts ponctuels ou rotationnels, dislocations) produite à la surface de la calotte polaire, donc en relation directe avec la température, se conserve en profondeur aussi longtemps que seul le grossissement des grains intervient. D'après Alley [8], la vitesse de croissance des grains dépend de la teneur en impuretés solubles d'origine marine, celle-ci étant bien corrélée au rapport isotopique.

L'évolution de la taille des grains le long de la carotte de Dome $\mathrm{C}$ est clairement mise en évidence par les photographies des lames minces montrées sur la figure 1. On peut aussi noter que le grossissement des grains, qui implique une réduction de l'énergie des joints de grains, est caractérisé par une structure équiaxe des grains.

D'après les données recueillies aussi bien au Groenland qu'en Antarctique, la vitesse de grossissement des grains passe de $10^{-17} \mathrm{~m}^{2} / \mathrm{s}$ à $-50{ }^{\circ} \mathrm{C}$ à $10^{-15} \mathrm{~m}^{2} / \mathrm{s}$ à $-10^{\circ} \mathrm{C}[14]$. Au-dessus de $-10^{\circ} \mathrm{C}$, cette vitesse augmente beaucoup plus vite que celle déduite de l'activation thermique à basse température ; à $0^{\circ} \mathrm{C}$, elle prend une valeur de plus de six ordres de grandeur supérieure à celle obtenue à $-10^{\circ} \mathrm{C}$. Un changement des mécanismes de diffusion des molécules d'eau près de la température de fusion est probable. Le grossissement des grains est observé tant que l'énergie de déformation garde une faible valeur par rapport à celle due à la courbe des joints de grains. A Vostok, le grossissement des grains est observé jusqu'à $2500 \mathrm{~m}$ de profondeur. Mais, l'énergie de déformation correspondant aux vitesses de déformation calculées au-dessus de $2500 \mathrm{~m}$ est très faible. A Byrd (Antarctique de l'Ouest), le grossissement des grains n'est plus observé en-dessous de $500 \mathrm{~m}$. Mais, l'accumulation annuelle et la température étant beaucoup plus élevées qu'à Vostok, les vitesses de déformation sont en surface plus de dix fois supérieures à celles déterminées à ce site.

\subsection{Recristallisation par rotation}

Dès que l'énergie de déformation devient suffisamment élevée pour bloquer le grossissement des grains, des sousgrains se forment et, progressivement, de nouveaux grains apparaissent. Cette polygonisation, qui correspond à un arrangement des dislocations dans des plans, se traduit par une désorientation du réseau cristallin observable visuellement en lumière polarisée. Les grains fraîchement nucléés grossissent sous l'effet de l'importante variation entre l'énergie de déformation de ces grains et celle des grains « anciens " encore chargés de dislocations. Les vitesses de migration des joints de grains correspondant à ce régime 
de recristallisation sont plus élevées que celles observées durant le grossissement «normal» des grains, la force motrice de cette migration étant plus élevée. Mais la mobilité intrinsèque des joints de grains garde la même valeur. La compétition entre la nucléation de nouveaux grains et le grossissement explique la faible variation de la taille des grains avec la profondeur observée, par exemple, dans la carotte de Byrd en-dessous de $500 \mathrm{~m}$ [3].

\subsection{Recristallisation par migration}

Une brusque variation de la texture de la glace de la carotte de Byrd a été observée vers $1800 \mathrm{~m}$ [3]. La taille des grains passe en quelques mètres de $20 \mathrm{~mm}^{2}$ à $200 \mathrm{~mm}^{2}$ et cette texture est préservée jusqu'au lit rocheux où la température de fusion de la glace est atteinte. Ce régime de recristallisation est donc associé à une vitesse de migration des joints de grains très élevée. Une variation importante de l'énergie de déformation sur quelques mètres n'étant pas envisageable, il faut donc admettre que la mobilité intrinsèque des joints de grains a changé d'une manière catastrophique vers $1800 \mathrm{~m}$. La température est le seul paramètre qui puisse être à l'origine d'une telle instabilité. En effet, ce régime de recristallisation n'a été observé qu'au dessus de $-10^{\circ} \mathrm{C}$ et la température corrigée de l'effet de la pression est proche de $-10^{\circ} \mathrm{C}$ à $1800 \mathrm{~m}$ à Byrd. La texture des glaces des glaciers tempérés est d'ailleurs tout à fait similaire à celle de la carotte de Byrd. Cette recristallisation, qui associe la nucléation de grains par gonflement de portions de joints de grains et leur grossissement, est celle que chacun peut reconnaître en observant la glace des glaciers tempérés. La structure équiaxe des grains est associée à la recristallisation par rotation alors qu'une structure de grains enchevêtrés est associée à la recristallisation par migration.

La déformation nécessaire à l'initiation de la recristallisation par migration ne dépasse pas $1 \%$; un cycle complet de recristallisation implique une déformation de l'ordre de $10 \%$ [14]. Cette rapide recristallisation s'oppose ainsi à tout effet de mémoire de l'histoire de la déformation; la texture (orientation préférentielle et taille des grains) est directement reliée à la contrainte. La taille des grains semble varier comme l'inverse de la contrainte [15].

\section{DÉFORMATION DE LA GLACE}

\subsection{Relations contrainte/vitesse de déformation pour les glaces froides}

La figure 2 montre les résultats d'essais mécaniques obtenus en laboratoire sur des glaces monocristallines et polycristallines. Les données concernent le fluage stationnaire pour le monocristal et le fluage minimum correspondant à une déformation proche de $1 \%$ pour les polycristaux. Les monocristaux étaient déformés de façon à initier le glissement sur les plans de base (plans [0001]). Les vitesses de déformation du monocristal sont plus de 1000 fois supérieures à celle du polycristal. Le résultat le plus discuté concerne l'évolution de la valeur de $n$ avec la contrainte pour le polycristal. Les tests n'ont été interrompus qu'après avoir atteint le fluage minimum, ce qui a nécessité des essais dépassant plusieurs mois. L'exposant

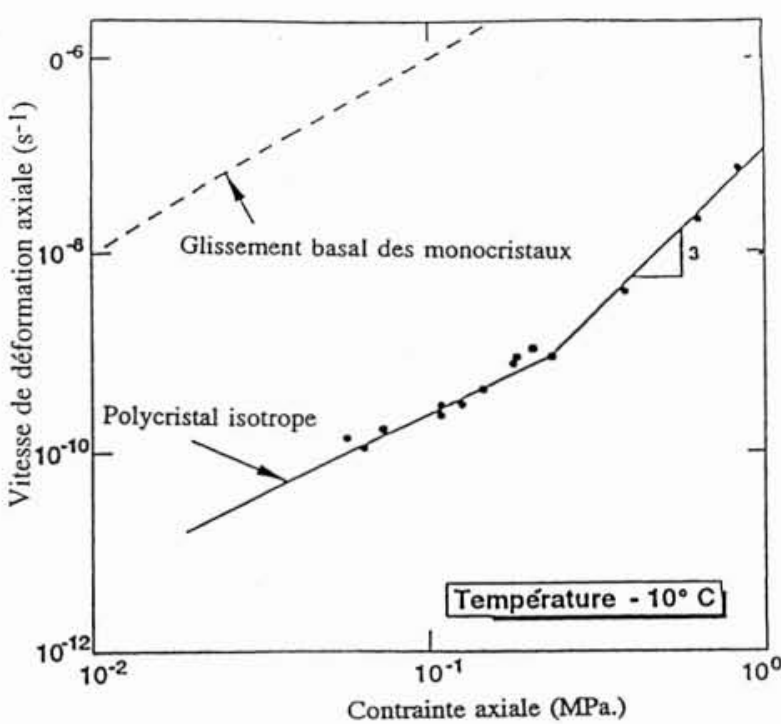

2. Variation de la vitesse de déformation minimum d'un polycristal isotrope avec la contrainte à $-10^{\circ} \mathrm{C}$. Les résultats sur le glissement basal du monocristal sont donnés à titre de comparaison.

est proche de trois pour une contrainte axiale supérieure à $0,2 \mathrm{MPa}$. Mais, dans les conditions qui prévalent dans les calottes polaires, cet exposant est inférieur à 2. Un comportement newtonien est d'ailleurs attendu pour les contraintes inférieures à $0,02 \mathrm{MPa}[1 ; 9]$.

L'étude de la texture des glaces des calottes polaires a permis de montrer que le glissement intracristallin par glissement des dislocations dans les plans de base était le mode de déformation prépondérant même près de la surface où les vitesses peuvent être inférieures à $10^{-13} \mathrm{~s}^{-1}$. En effet, l'orientation préférentielle des cristaux qui se développe depuis la surface n'est pas compatible avec le glissement aux joints de grains ou le fluage diffusionnel $[3 ; 5]$. La valeur de $n=3$ est expliquée par la montée des dislocations hors du plan de base ou par le glissement des dislocations dans les plans prismatiques ou pyramidaux [14]. Ces modes de déformation permettraient une partielle accommodation du glissement basal. En effet, la déformation du polycristal nécessite au moins quatre systèmes de déformation [16], le glissement basal ou la montée n'en fournissant que deux. La difficulté d'initier le glissement hors du plan de base explique l'important décalage entre les vitesses du fluage du monocristal et du polycristal. Parallèlement, cette anisotropie plastique du monocristal est à l'origine du développement d'un champ de contraintes internes dans le polycristal durant le fluage transitoire [14]. A la mise en charge d'un échantillon, l'état de contrainte à l'échelle du grain est uniforme. Du fait de la faible résistance au cisaillement dans les plans de base, la contrainte dans ces plans se relaxe et la charge est transférée aux plans prismatiques et pyramidaux.

\subsection{Modélisation du comportement mécanique du po- lycristal}

Dans le but de déduire la loi de comportement d'une glace d'un glacier froid dont l'orientation préférentielle des cristaux est connue, diverses approches micromécaniques ont 
été proposées [9;17-19]. Ces approches s'appuient sur une méthode d'homogénéisation qui permet d'exprimer les grandeurs globales (à l'échelle du polycristal) comme la moyenne des grandeurs locales (à l'échelle du monocristal). Ainsi, le comportement mécanique d'un polycristal est déduit du comportement de chacun de ses grains. L'hypothèse la plus souvent faite consiste à admettre que la cission locale sur les plans de base est proportionnelle à la contrainte macroscopique sur ces plans et que la déformation est produite seulement par le glissement basal.

Une méthode d'homogénéisation plus élaborée, dite auto-cohérente, qui satisfait aux conditions d'équilibre des contraintes et de compatibilité des déformations à l'intérieur du polycristal, a été adaptée à la glace. Ce modèle a été développé pour décrire le comportement de matériaux cristallins anisotropes et l'évolution de cette anisotropie avec la déformation [20]. Au moins quatre systèmes de déformation doivent être activés, soit, pour la glace, le glissement basal et le glissement prismatique (ou pyramidal). Avec cette formulation, l'état local (déformations et contraintes) peut être différent de l'état macroscopique (à l'échelle du polycristal). Dans ce modèle, chaque grain est considéré comme une inclusion à l'intérieur d'un milieu homogène équivalent (HEM) dont le comportement est celui du polycristal. Ce traitement aboutit à une équation d'interaction qui relie les contraintes et déformations locales aux variables macroscopiques du milieu équivalent. Cette approche impose que la moyenne des contraintes (et déformations) sur tous les grains correspondent à la contrainte (et déformation) à l'échelle du polycristal.

La figure 3 montre la variation de la contrainte et de la déformation à l'échelle du grain avec l'angle entre la direction de la contrainte de compression et la normale aux plans de base pour une glace isotrope. Cette simulation a été faite pour une vitesse imposée de $10^{-7} \mathrm{~s}^{-1}$ à $-10^{\circ} \mathrm{C}$. L'accord avec les données de laboratoire a été obtenu par l'approche auto-cohérente en fixant la résistance au glissement dans les plans prismatiques vingt fois plus forte que celle dans les plans de base. Les modèles limites (à contraintes et déformations homogènes dans le polycristal) ne peuvent caractériser le comportement mécanique de la glace. Le modèle statique ( $\sigma=$ cte $)$ ne peut prédire des contraintes suffisantes, alors que le modèle de Taylor ( $\varepsilon=$ cte ) impose des contraintes beaucoup trop élevées (fig. 3). D'autre part, d'après le modèle auto-cohérent, le glissement dans les plans de base contribue à plus de $90 \%$ de la déformation du polycristal, ce qui est compatible avec la structure et la dynamique des dislocations dans la glace.

En grandes déformations, ces approches permettent de prédire l'évolution de la texture d'un polycristal de glace au cours de son histoire thermo-mécanique. Cependant, un résultat réaliste ne pourra être obtenu qu'en prenant en compte la recristallisation. Ceci est possible en introduisant un degré de liberté supplémentaire (la migration des joints de grains) dans la loi d'interaction du polycristal.

\subsection{Rôle de l'eau dans le fluage de la glace tempérée}

La teneur en eau liquide de la glace des glaciers tempérés est typiquement de l'ordre de $1 \%$ [21]. Cette eau se localise généralement dans des veines à la jonction de trois ou quatre grains; mais, sous l'effet de contraintes non hydrostatiques, elle peut former des lentilles aux jonctions de deux grains [22]. Des données précises sur la localisation
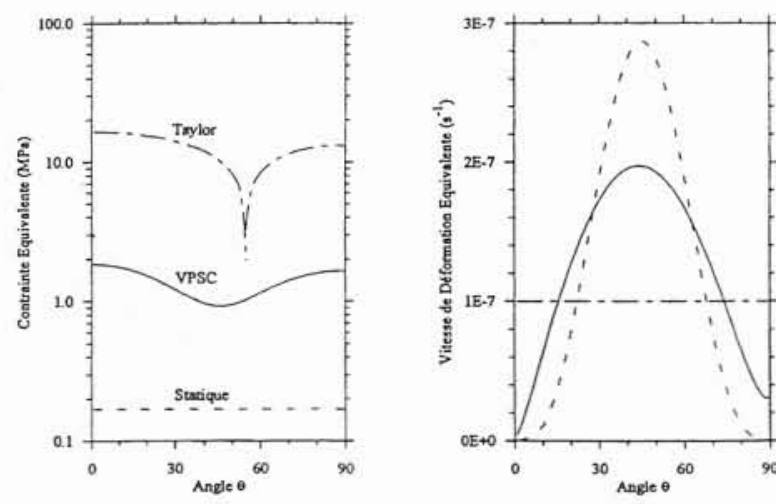

3. Variation de la contrainte et vitesse de déformation équivalente en fonction de l'orientation du grain par rapport à la direction de l'axe de compression; les courbes concernent le modèle auto-cohérent (VPSC), le modèle de Taylor (déformations homogènes) et le modèle statique (contraintes homogènes). Les calculs ont été réalisés pour une vitesse de déformation imposée au polycristal égale à $10^{-7} \mathrm{~s}^{-1}$.

de l'eau dans la glace active des glaciers sont toujours attendues pour comprendre le rôle de cette phase dans le comportement mécanique. Il faut prendre en considération le fait que cette glace tempérée recristallise sous un régime de vitesses de migration des joints de grains très élevées.

La dépendance de la viscosité de la glace avec la teneur en eau a été démontrée par Duval [23]. L'action de l'eau sur le fluage de la glace est probablement complexe. Tout d'abord, l'eau doit atténuer le champ de contraintes internes dans le polycristal, qui résulte de l'incompatibilité de déformation entre les grains d'orientations différentes. L'eau peut aussi, par une modification des processus de diffusion, être à l'origine de la mobilité accrue des joints de grains à la température de fusion. Enfin, comme le suggère Lliboutry [22], des processus de fusion et regel au niveau des joints de grains peuvent contribuer à la déformation de la glace. Il faut, cependant, noter que les mécanismes qui contrôlent la vitesse du fluage sont inchangés. En effet, l'exposant $n$ prend les mêmes valeurs en dessous et à la température de fusion.

L'intérêt de cette analyse du comportement mécanique de ce matériau biphasé dépasse la glaciologie. Le comportement des roches partiellement liquides du manteau terrestre fait l'objet depuis quelques années d'un grand nombre d'études [24]. La mise en forme des matériaux superplastiques ou le frittage des céramiques est largement facilité par la présence d'un film liquide aux joints de grains [25]. La glace des glaciers tempérés, dont le comportement mécanique peut être appréhendé sans grandes difficultés aussi bien en laboratoire que sur le terrain, est un matériau modèle dont la promotion doit être faite.

\section{Remerciements}

Ce travail a été financé par le PNEDC (Programme National d'Etudes de la Dynamique du Climat) de l'INSU/CNRS, le Département Sciences Pour l'Ingénieur du CNRS et la Commission des Communautés Européennes par le programme «Environnement». 


\section{O. CASTELNAU, P. DUVAL}

\section{Références}

[1] Pimienta P. et Duval. P. (1987). - Rate controlling processes in the creep of polar glacier ice, Journal de Physique, tome 48 , pp. C1243-C1248.

[2] Lipenkov V. Ya. and SAlamatin A.N. - Bubbly ice densification in ice sheets : applications (soumis à : Journal of Geophysical Research).

[3] Gow A.J. and Williamson T. (1976). - Rheological implications of the internal structure and crystal fabrics of the West Antarctic ice sheet as revealed by deep core drilling at Byrd Station, CRREL Report 76-35.

[4] Herron S.I., Langway C.C. and Brugger K.A. (1985). Ultrasonic velocities and crystalline anisotropy in the ice core from Dye 3, Greenland, Geophysical Monograph 33, pp. 23-31.

[5] Lipenkov V. Ya., Barkov N.I., Duval P. and Pimienta P. (1989). - Crystalline texture of the $2083 \mathrm{~m}$ ice core at Vostok Station, Journal of Glaciology, 35, pp. 392-398.

[6] Pimienta P., Duval P. and Lipenkov V. Ya. (1987). Mechanical behavior of anisotropic polar ice, IAHS Publ. no. 170 , pp. $57-66$.

[7] PIMIENTA P. and Duval P. (1989). - Rheology of polar glacier ice, Annals of Glaciology, 12, pp. 206.

[8] Alley R.B. (1992), - Flow law hypotheses for ice-sheet modeling, Journal of Glaciology, 38, pp. 245-255.

[9] LliboutRY L. and DUVAL P. (1985). - Various isotropic and anisotropic ices found in glaciers and polar ice caps and their corresponding rheologies, Annales Geophysicae, 3. pp. 207-223.

[10] NYE J.F. and MAE S. (1972). - The effect of non-hydrostatic stress on intergranular water veins and lenses in ice, Journal of Glaciology, 11, pp. 81-101.

[11] Lliboutry L. (1976). - Physical processes in temperate glacier, Journal of Glaciology, 16, pp. 151-158.

[12] Lliboutry L. (1993). - Internal melting and ice accretion at the bottom of temperature glaciers, Journal of Glaciology, 39 , pp. $50-64$.
[13] PetrT J.R., DuVal. P. and Lorius C. (1987), - Long-term climatic changes indicated by crystal growth in polar ice. Nature, 326, pp. 62-64.

[14] Duval P., Ashby M.F. and Anderman I. (1983), - Ratecontrolling processes in the creep of polycrystalline ice, the Journal of Physical Chemistry, 87, pp. 4066-4074.

[15] JACKA T.H. and LI JUN 1995. - The steady-state crystal size of deforming ice, Annals of Glaciology, 20, pp. 13-18.

[16] Hutchinson J.W. (1977). - Creep and plasticity of hexagonal polycrystals as related to single crystal slip. Metal. Transactions, 8A, pp. 1465-1469.

[17] AZUMA N. and HigasHi A. (1985), - Formation processes of ice fabric pattern in ice sheets, Annals of Glaciology, 6. pp. 130-134.

[18] VAN DER VEen C.J. and Whill.ans I.M. (1994), - Development of fabric in ice, Cold Regions Science and Technology, 22, pp. 171-195.

[19] Castelnau O. and Duval P. (1995). - Simulations of anisotropy and fabric development. Annals of Glaciology, 20 , pp. $227-282$.

[20] Lebensohn R.A. and Tomé C.N. (1993). - A selfconsistent anisotropic approach for the simulation of plastic deformation and texture development: application to zirconium alloys, Acta Metall. Mater., 41, pp. 2611-2624.

[21] VAllon M., PettT J.R. and Fabre B. (1976). - Study of an ice core to the bedrock in the accumulation zone of an alpine glacier, Journal of Glaciology, 17, pp. 13-28.

[22] LLiBouTRY L. (1987). - Very slow flows of solids, Martinus Nijhoff publishers, $510 \mathrm{p}$.

[23] DuVAL P. (1977), - The role of the water content on the creep rate of polycrystalline ice, IAHS Publ., 118, pp. 29-33.

[24] Zhen-Ming Jin, Green H.W. and Zhou YI (1994), - Melt topology in partially molten mantle peridotite during ductile deformation. Nature, 372, pp. 164-166.

[25] Baudelet B., Dang M.C. and Bordeaux F. (1992), Mechanical behavior of an aluminium alloy with fusible grain boundaries, Scripta Met. et Mat., 26, pp. 573-577. 